\title{
Motorists' voluntary yielding of right of way at uncontrolled midblock crosswalks with rectangular rapid flashing beacons
}

\section{Authors: Ahmed Al-Kaisy, Guilherme T. Miyake, Joey Staszcuk, and Danielle Scharf}

This is an Accepted Manuscript of an article published in Journal of Transportation Safety \& Security on July 2018, available online: https://www.tandfonline.com/10.1080/19439962.2016.1267827.

Al-Kaisy, Ahmed, Guilherme T. Miyake, Joey Staszcuk, and Danielle Scharf. "Motorists' voluntary yielding of right of way at uncontrolled midblock crosswalks with rectangular rapid flashing beacons." Journal of Transportation Safety \& Security 10, no. 4 (July 2018): 303-317. DOI:

10.1080/19439962.2016.1267827. 


\title{
Motorists' Voluntary Yielding of Right of Way at Uncontrolled Mid-Block Crosswalks with Rectangular Rapid Flashing Beacons
}

\author{
Ahmed Al-Kaisy, Ph.D., P.E. (corresponding author) \\ Professor, Civil Engineering Department \\ Montana State University \\ PO Box 174250 \\ Bozeman, MT 59717 \\ Phone: (406) 994-6116 \\ Fax: (406) 994-6105 \\ aalkaisy@coe.montana.edu \\ Guilherme T. Miyake \\ Undergraduate Research Assistant \\ Montana State University \\ Bozeman, MT 59717 \\ Email: guilherme.t.miyake@gmail.com \\ Joey Staszcuk, M.Sc., P.E. \\ Transportation Engineer \\ Sanderson Stewart \\ Bozeman, MT 59715 \\ Phone: (406) 522-9876 \\ Email: jstaszcuk@sandersonstewart.com \\ Danielle Scharf, M.Sc., P.E., PTOE \\ Associate Principal and Branch Manager \\ Sanderson Stewart \\ Bozeman, MT 59715 \\ Phone: (406) 522-9876 \\ Email: dscharf@sandersonstewart.com
}




\begin{abstract}
This paper presents an investigation into the motorists' voluntary yielding behavior to bicycles and pedestrians at uncontrolled mid-block crosswalk locations. Two study sites in the state of Montana with LED rectangular rapid flashing beacons (RRFB) warning devices were used in this investigation. Video records were acquired at the two study sites and video data was reduced to investigate the motorist's voluntary yielding of right of way to pedestrians and bicycles as they approach the crosswalks. The study found that the motorists' voluntary yielding increased with the activation of the RRFB devices at the two study sites. This finding is very consistent with the few recent studies that reported increase in the overall yielding behavior associated with the use of those devices. The study also found that the waiting position of crosswalk user(s), the presence of children and elderly, the number of crosswalk users and peak periods are all important factors that affect the voluntary yielding behavior of motorists. Study results suggest that the visibility of crosswalk users by motorists is very important consideration in the selection of crosswalk location. Further, the study recommends the push-button RRFB devices be located as close as practically possible to the roadway for increased device activation and motorists' voluntary yielding.
\end{abstract}

Keywords: $\quad$ crosswalk, flashing beacons, pedestrians, bicycles, right of way, yielding 


\section{INTRODUCTION}

The use of alternative modes of transport, mainly biking and walking, is critical in promoting sustainable transportation systems in the US and in other parts of the world. At the national level, most highway agencies and municipalities have taken steps to incorporate these two modes in the planning and design of new transportation projects and in retrofitting existing facilities. However, if these modes were to be successfully integrated in the highway system, the safety of pedestrians and bicyclists has to be carefully thought of throughout the process. Despite the general decrease in pedestrian and cyclist casualties over the past two decades, they are still overrepresented in our national crash statistics. In 2012, 4,743 pedestrians died and more than 76,000 pedestrians were injured in motor vehicle crashes (NHTSA 2014a). During the same year, 726 pedalcyclists were killed and an additional 49,000 were injured in motor vehicle traffic crashes (NHTSA 2014b).

Using data collected from six states, Hunter et al. found that one fourth of pedestrian-vehicle crashes occur at uncontrolled midblock locations (Hunter et al. 1996). A study by Zegeer et al. (2005) revealed that on two-lane roads, the presence of a marked crosswalk alone at an uncontrolled location was associated with no difference in pedestrian crash rate, compared to an unmarked crosswalk. Further, on multilane roads with traffic volumes above 12,000 vehicles per day, having a marked crosswalk alone (with no additional pedestrian treatments) was associated with a higher pedestrian crash rate than comparable roadways without marked crossings. The study recommended more substantial measures to provide for safer pedestrian crossings such as adding traffic signals with pedestrian signals, providing raised medians, and speed-reducing measures among other recommended measures.

To improve the safety of pedestrians at uncontrolled crosswalk locations, several techniques and technologies have been used to increase driver yielding to pedestrians at those locations. Older techniques include the use of flashing overhead and side-mounted standard yellow beacons (Ellis et al. 2007) and in-street "YIELD TO PEDESTRIAN" portable signs and in-roadway lights (Prevedouros 2001). A more recent and effective technique is the pedestrian crossing device that employs light-emitting diode (LED) rectangular rapid-flashing beacons (RRFB) that are similar in operation to emergency flashers on police vehicles (Shurbutt and Van Houten 2010). The latter device is the focus of the current study.

The effectiveness of the RRFB in increasing the yielding of motorists to crosswalk users has been investigated in a few recent studies (Shurbutt and Van Houten 2010, Hunter et al. 2012, Foster et al. 2014, Hunter-Zaworski and Mueller 2012, Ross et al. 2012). Shurbutt and Van Houten (2010) conducted a before-after study of RRFB devices at pedestrian midblock crossings in Saint Petersburg, Florida. The study found that yielding by motorists had remarkably increased from $18 \%$ at baseline to $80 \%$ or more depending on the number of devices installed on the crossing. Similar results were reported in another study by Hunter-Zaworski and Mueller (2012) in investigating the RRFB's effectiveness at multiple sites in Oregon. Another study by Hunter et al. (2012) found that motorists' yielding to crosswalk users increased from $2 \%$ before 
to $54 \%$ after installation when the device was activated. Significant increase in motorists' yielding to bicycles and pedestrians were also reported in earlier studies (Foster et al. 2014, Hunter-Zaworski and Mueller 2012). The majority of those studies included comparisons before and after the installation of the RRFB devices. Two important issues that have to be treated carefully in such investigations are: 1) the temporal effect of the newly installed devices that may change over time, and 2) the effect of other changes in signs and markings that may be done simultaneously with the RRFB new installations.

\section{STUDY AIMS}

In all previous studies, the yielding of motorists was defined in the context of slowing down or stopping to give way to someone who's waiting to use the crosswalk (voluntary) as well as yielding to those already in the crosswalk in a conflict avoidance maneuver (mandatory). Almost all traffic laws and regulations without exception require the driver of a vehicle to yield the right of way (slowing down or stopping if need be) to a pedestrian or a bicyclist crossing the roadway within a crosswalk on the roadway upon which the vehicle is traveling. This situation was described above as mandatory yielding. On the other hand, the obligation to yield to pedestrians or bicyclists who are not physically on the crosswalk (waiting beyond the curb) is not very clear to most drivers though they are strongly encouraged to do so. Of course, it should go without saying that, in practice, a vehicle should yield to a pedestrian regardless of a statutory obligation to do so. This latter situation is described above as "voluntary" yielding.

The current study is only concerned with the motorists' voluntary yielding of right of way to pedestrians and bicycles in conjunction with the use of the RRFB devices. Voluntary yielding is very important indicator of the motorists' willingness to give up the right of way to the more vulnerable users, bicycles and pedestrians, at those points of conflict. The aim of the current research was to investigate the RRFB effectiveness in increasing the motorists' voluntary yielding to pedestrians and bicyclists. The other aim of the study is to examine the factors and circumstances that would influence the motorists' voluntary yielding behavior at uncontrolled mid-block crossing locations.

\section{STUDY DESIGN}

\subsection{Data Collection}

Two mid-block crosswalk locations with RRFB devices were used as study sites in this investigation. The RRFB devices were already installed at these sites for a long period prior to the study, so the temporal effect on the RRFB effectiveness was not a concern. Portable video data collection devices were installed at the study sites so that the video footage would capture the prospective pedestrians/bicyclists at the crosswalk, the push-button device, and enough distance along the roadway to clearly show vehicles as they approach the crosswalk location. 
The video cameras were relatively small in size and remained largely invisible to drivers and crosswalk users. Figure 1 shows the video data collection equipment deployed in the field.

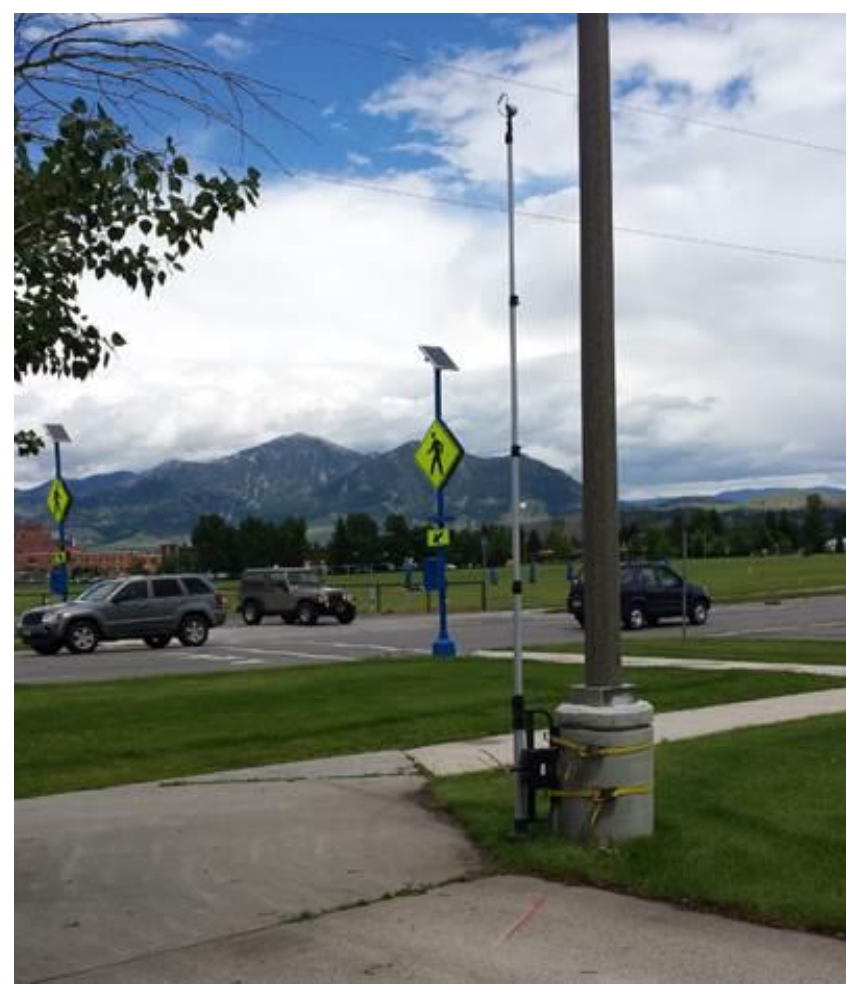

FIGURE 1 Video data collection equipment attached to a utility mast.

\subsection{Study Variables}

The current study investigated several variables that are expected or thought to affect the motorists' voluntary yielding behavior at mid-block crosswalk locations. Besides the activation of the RRFB device, those variables are mostly related to the characteristics of crosswalk users and are listed below:

I. Crosswalk user type (pedestrians and cyclists)

II. Presence of children and elderly

III. Crosswalk user waiting position

IV. Number of crosswalk users (waiting to cross in a single crossing maneuver)

For analysis purposes, the study also gathered information on the lane on which the vehicle is traveling and the direction of the crossing maneuver.

\subsection{Data Reduction Protocols}


One of the challenging issues in the current research is to develop a sound data reduction strategy that would clearly identify situations which call for motorists' voluntary yielding of right of way, while excluding conflict avoidance maneuvers. A major determinant of driver's yielding behavior in a crossing situation is the distance between the vehicle and the crosswalk. Two situations had to be addressed carefully in reducing video data to make sure that the driver has a choice (to yield or proceed) in responding to waiting pedestrians and bicyclists at the crosswalk location.

I. If the vehicle is too close to the crosswalk, then it may not be practical for the driver to stop the vehicle safely at the crosswalk, and thus he/she may not have the choice to yield to crosswalk users. Data reduction protocols had to exclude those instances using stopping distance requirements for vehicles approaching the crosswalk location. It is expected that different vehicles and drivers tend to apply different deceleration rates in a stopping situation. To apply a systematic approach in processing video records, a certain distance upstream of the crosswalk has to be designated and vehicles within this distance will not be further analyzed in this study. With the objective of excluding primarily the vehicles that are reasonably close to the crosswalk, two decisions were made in determining that distance. The first was to use the mean deceleration rate of $17.7 \mathrm{ft} / \mathrm{s}^{2}$ (Donnell at al. 2012) instead of the $90^{\text {th }}$ percentile rate of $11.2 \mathrm{ft} / \mathrm{s}^{2}$ in calculating the stopping distance (AASHTO 2011). This was deemed more appropriate given that video data was collected during the summer with dry pavement conditions. The other decision made in calculating the distance was to disregard the reaction distance (or perception-reaction time). The rationale for the latter decision is that in both study sites, ample visibility exists that would enable the motorist to observe the pedestrians and cyclists as they approach their waiting position in anticipation of performing their intended crossing maneuver. In other words, observing crosswalk users and their intended maneuver takes place prior to motorists having to decide on yielding to crosswalk users. These two decisions, which were made upon watching extensive video records, aimed to avoid unnecessarily excluding large proportion of drivers who have the choice to yield or proceed without ending up in a conflict with pedestrians or bicycles.

II. If the vehicle is relatively far from the crosswalk, then the driver may not perceive the need to stop (or slow down) to allow crosswalk users perform their intended crossing maneuver. In this situation, the driver may slow down or proceed at his/her original speed, depending on the distance from the crosswalk, without interfering with its use. Addressing this situation was relatively straightforward. If the pedestrians and/or bikers decided to use the crosswalk and the vehicle did not have to stop, then that event was excluded from the data due to lack of conflict and the fact that this research is more concerned about the driver yielding behavior should a crosswalk user is waiting to cross the roadway (i.e. voluntary yielding). 
All events where the motorists had to stop or slow down to avoid a conflict with sidewalk users (already in the crossing) were excluded from the analysis.

\subsection{Study Sites}

Two study sites in the state of Montana were used in this research. A brief description of these sites is presented next.

Study site \#1: This site is located at King Avenue (West) which is a four-lane divided major urban arterial in the western part of the City of Billings, Montana. The site is a mid-block crossing that has a ladder style crosswalk with yield line and a push-button LED RRFB system. The Annual Average Daily Traffic (AADT) at this section of King Avenue is estimated at around 15000 vehicles per day (vpd) in 2013 and the speed limit at this section is $35 \mathrm{mph}$. A total of 109 hours of video recordings in the two directions of travel (eastbound and westbound) were used in this study, which resulted in 276 crossing events that were eligible for data reduction in this study (i.e. with voluntary yielding). It is important to note that this site has two sets of separate RRFB devices (one in each direction) and that each observation represents a crossing of one direction of the road. The video data at this site was collected in July 2012 during daytime hours.

Study site \#2: This site is located at Kagy Boulvard which is a two-lane undivided urban arterial that runs east-west in the southern part of the City of Bozeman. The study site is a mid-block crossing at the southern part of the Montana State University (MSU) Campus with push-button LED RRFB devices on both sides of the crossing. The crossing is intended for pedestrian and bicycle traffic between the main campus buildings north of Kagy and the stadium and the few research and office buildings located south of Kagy at the fringe of the MSU campus. The AADT at this section of Kagy Boulevard is estimated at around 13000 vpd in 2013 and the speed limit is $35 \mathrm{mph}$. A total of 91 hours of video recording was acquired at this site for traffic in the westbound direction over more than a week in June 2014 during daytime. This has resulted in a total of 104 crossing events that met the data reduction protocols described earlier.

Figure 2 shows images of the two sites extracted from Google Earth street view shots.

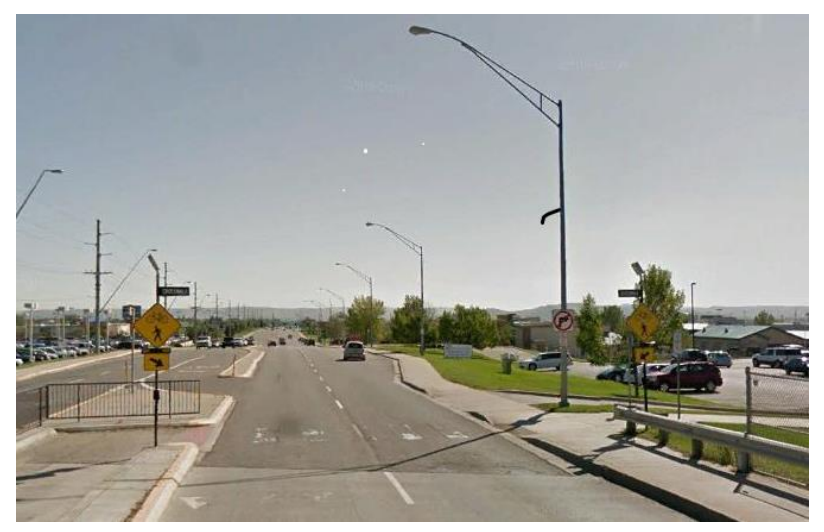

(a)

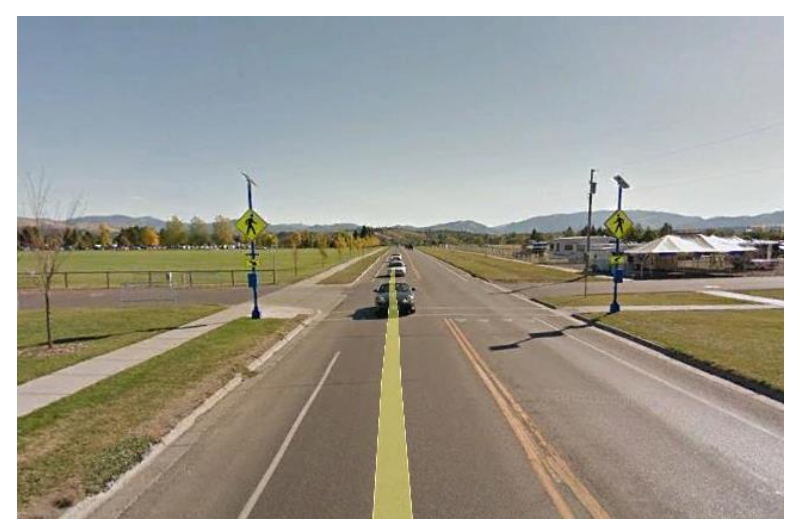

(b)

Figure 2: Street view of the two study sites a) King Avenue eastbound, b) Kagy Boulevard (Source: Google Earth) 


\section{ANALYSIS OF RESULTS}

In this section, the motorists' voluntary yielding to pedestrians and bicycles will be analyzed using study variables that were discussed earlier in study design.

\subsection{General}

The use of the push-button RRFB device is expected to increase the tendency of drivers to yield to pedestrians and other crosswalk users in general as reported in recent studies. This effect is expected to also include motorists' voluntary yielding described earlier which is the focus of this study. Table 1 provides a summary of the observations at the two study sites with and without RRFB device activation and the motorists' voluntary yielding behavior. As shown in this table, the total number of observations at King Avenue site is 276 of which 109 are associated with voluntary yielding, or around $39.5 \%$ of the total observations. Corresponding numbers from the Kagy Boulevard site are 104 total observations of which 82 were associated with voluntary yielding behavior. This results in a voluntary yielding rate of $78.8 \%$, which is almost twice as high as the rate at King Avenue site. One explanation for the vast disparity between the two rates is that the Kagy Boulevard site is located at the fringe of a university campus where drivers are expected to yield voluntarily to student pedestrians and cyclists waiting to cross at the crosswalk. The same level of expectation does not exist at the King Avenue site that is located primarily in a commercial district. Finally, another factor that may have contributed to this disparity in rates is the higher percentage of RRFB device activation at the Kagy Boulevard site, to be discussed later.

In regards to the status of the RRFB activation, the rate of voluntary yielding when the RRFB device was activated at the King Avenue site was 45.2\% versus 31.9\% when the RRFB was not activated. Corresponding rates from Kagy Boulevard site were 84.5\% and 55\% respectively. These numbers are consistent with the findings from other recent studies about the effectiveness of the RRFB devices in increasing the motorists' yielding behavior. The Pearson's chi-square results indicate that the use of the RRFB device is associated with the difference in motorists' voluntary yielding behavior (i.e. difference was not due to chance) as suggested by the small Pvalues at the two study sites.

TABLE 1 Voluntary Yielding Behavior at the Two Study Sites

\begin{tabular}{|l|c|c|c|c|c|c|c|c|}
\hline \multirow{2}{*}{ Study Site } & \multicolumn{4}{|c|}{ King Avenue } & \multicolumn{4}{c|}{ Kagy Boulevard } \\
\cline { 2 - 9 } & $\begin{array}{c}\text { Lights } \\
\text { Activated }\end{array}$ & $\begin{array}{c}\text { Lights } \\
\text { Off }\end{array}$ & Total & $\begin{array}{c}\text { Pearson } \\
\chi^{2}\end{array}$ & $\begin{array}{c}\text { Lights } \\
\text { Activated }\end{array}$ & $\begin{array}{c}\text { Lights } \\
\text { Off }\end{array}$ & Total & $\begin{array}{c}\text { Pearson } \\
\chi^{2}\end{array}$ \\
\hline Total Observations & 157 & 119 & 276 & & 84 & 20 & 104 & \\
Yielding & 71 & 38 & 109 & $\mathrm{P}=0.025$ & 74 & 11 & 82 & $\mathrm{P}<0.001$ \\
Percentage & $45.2 \%$ & $31.9 \%$ & $39.5 \%$ & & $84.5 \%$ & $55.0 \%$ & $78.8 \%$ & \\
\hline
\end{tabular}

A further note on device activation rate is that the higher percentage of RRFB activations at Kagy Boulevard may primarily be due to the placement of the device. Specifically, at the Kagy 
Boulevard site, the device stands close to the curb and within easy reach from the crosswalk user without imposing any change in his/her trajectory. On the other hand, at the King Avenue site, the device stands away from the curb and may require change in the crosswalk users' trajectory, which may discourage some users from activating the device.

\subsection{User Waiting Position}

Another hypothesis that was tested in this study is that the closer the pedestrian and/or other users from the curb, the more likely the drivers to yield as they approach the crosswalk. In this study, distance from the curb was described in three different ways: "at curb" if the user is waiting immediately at the curb, "off curb" if the user is waiting at some distance back from the curb but before the push button device, and "other" to refer to the few instances when users would wait away from the curb and beyond the push-button device. Figure 3 shows the percentage of voluntary yield observations as a function of the distance from the curb both with and without RRFB device activation at the two study sites. Those numbers as well as the number of observations used in the analysis are also provided in Table 2.

At the King Avenue site, it is clear that the user waiting position has an important effect on the voluntary yielding behavior of the motorists overall. The effect of RRFB activation shows a logical pattern for the three different distance categories except for cases when the users were waiting immediately "at the curb." In those cases, the activation of the RRFB device was associated with lower percentage of yield cases which is unexpected and inconsistent with the general trend discussed earlier in the previous section. At the Kagy Boulevard site, 97 observation were "at curb", 7 "off curb" and no observation under "other" category. The very small number of observations for the "off curb" category is largely behind the fact that the voluntary yielding rate for "off curb" is higher than the corresponding rate for the "at curb" category, and as such results should be interpreted with caution.

Table 2 Voluntary Yielding Rate as a Function of User Waiting Position at the Two Study Sites

\begin{tabular}{|c|c|c|c|c|c|c|}
\hline \multicolumn{7}{|c|}{ King Avenue } \\
\hline Lights Activated & \multicolumn{3}{|c|}{ Yes } & \multicolumn{3}{|c|}{ No } \\
\hline Waiting Position & Yielding & Total & Percentage & Yielding & Total & Percentage \\
\hline At curb & 39 & 65 & $60.0 \%$ & 22 & 28 & $78.6 \%$ \\
\hline Off curb & 27 & 69 & $39.1 \%$ & 10 & 52 & $19.2 \%$ \\
\hline Other & 5 & 23 & $21.7 \%$ & 6 & 39 & $15.4 \%$ \\
\hline \multicolumn{7}{|c|}{ Kagy Boulevard } \\
\hline Lights Activated & \multicolumn{3}{|c|}{ Yes } & \multicolumn{3}{|c|}{ No } \\
\hline Waiting Position & Yielding & Total & Percentage & Yielding & Total & Percentage \\
\hline At curb & 69 & 82 & $84.1 \%$ & 11 & 15 & $73.3 \%$ \\
\hline Off curb & 2 & 2 & $100.0 \%$ & 0 & 5 & $0.0 \%$ \\
\hline Other & 0 & 0 & $0.0 \%$ & 0 & 0 & $0.0 \%$ \\
\hline
\end{tabular}




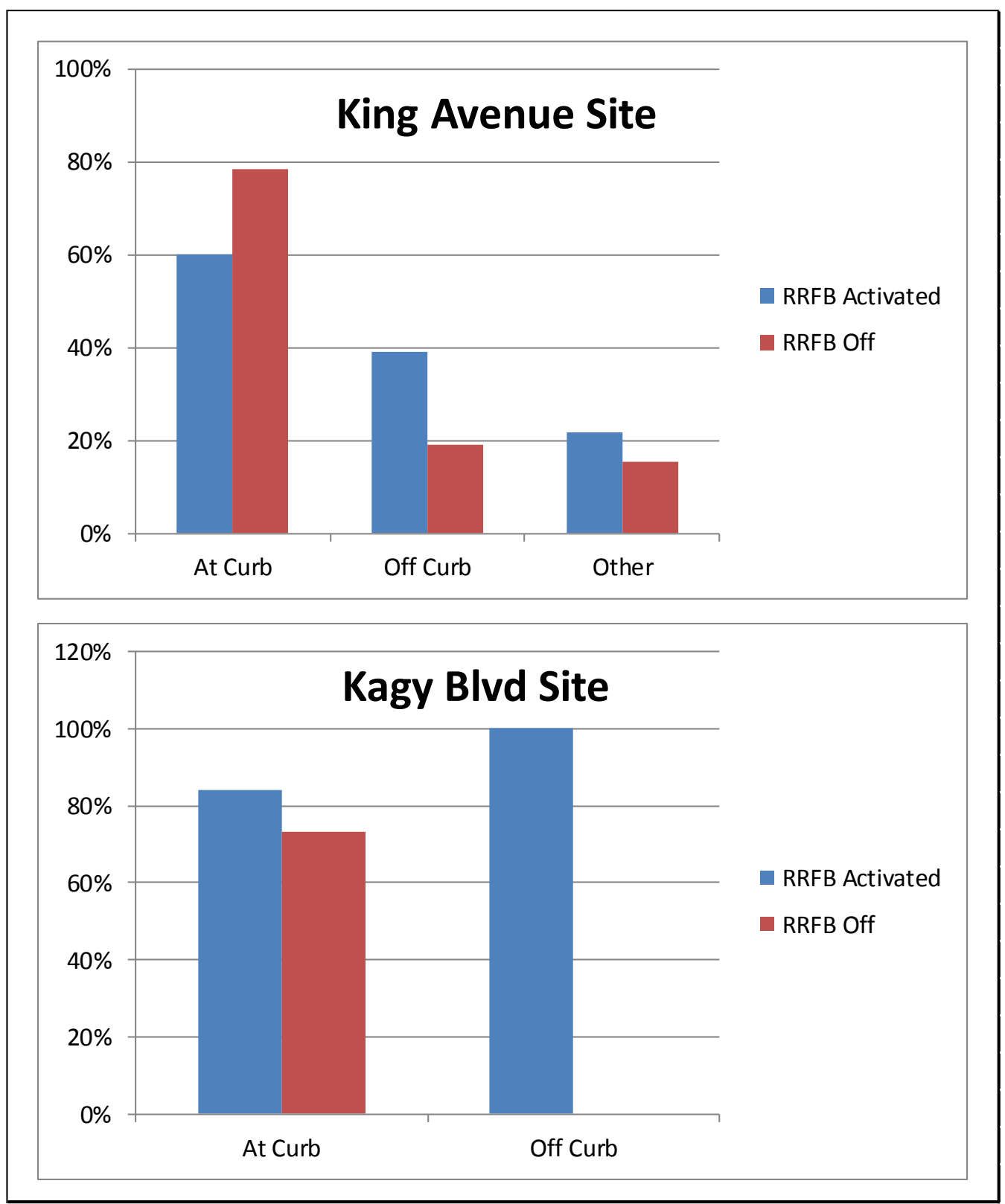

FIGURE 3 Voluntary yielding rates as a function of user waiting position and RRFB activation status.

\subsection{Children and Elderly Users}

One of the research questions that the current study attempts to answer is whether drivers are more likely to voluntarily yield if the users waiting to use the crosswalk are children or elderly. A total of 37 and 15 observations at King Avenue and Kagy Boulevard sites respectively were identified, in which at least one child, one elder or both were among the crosswalk users. Results are presented in Table $\underline{3} z$ below. 
TABLE $\underline{3} z$ Motorists' Voluntary Yielding in the Presence of Children and Elderly

\begin{tabular}{|l|c|c|c|c|c|c|}
\hline \multirow{2}{*}{$\begin{array}{c}\text { Presence of Child or } \\
\text { Elder }\end{array}$} & \multicolumn{3}{|c|}{ King Avenue } & \multicolumn{3}{c|}{ Kagy Boulevard } \\
\cline { 2 - 7 } & Yes & No & Pearson $\chi^{2}$ & Yes & No & Pearson $\chi^{2}$ \\
\hline Total Observations & 37 & 239 & & 15 & 89 & \\
Yielding & 22 & 87 & $\mathrm{P}=0.007$ & 15 & 67 & $\mathrm{P}=0.0302$ \\
Percentage & $59.5 \%$ & $36.4 \%$ & & $100.0 \%$ & $75.3 \%$ & \\
\hline
\end{tabular}

At a glance, this table clearly shows that the presence of children or elderly is associated with higher rate of voluntary yielding at the two study sites. At King Avenue study site, 22 out of 37 observations (60\% rate) were associated with yielding behavior, while at the Kagy Boulevard site, 15 out of 15 observations (100\% rate) were associated with voluntary yielding behavior. Those rates are notably higher that the yielding rates in the absence of children and elderly at the two study sites (36.4\% and $75.3 \%$ at the King and Kagy sites respectively). The Pearson's chisquare results indicate that the presence of children or elderly is associated with the motorists' voluntary yielding behavior at the two study sites at $95 \%$ confidence level. Further, it was of interest to know how the presence of children's and elders affected the drivers yielding behavior with and without the RRFB device activation. Table $\underline{4} z$ summarizes the results of this investigation. At King Avenue site, with the larger number of observations, the percentage of drivers yielding to pedestrians was slightly higher when the device was not activated. However, the difference in percentages is relatively small (in the order of 6\%) and therefore it cannot serve as an evidence of change in drivers' behavior. At the Kagy Boulevard site, the percentage of drivers yielding was $100 \%$ with and without RRFB activation, however, the number of observations was relatively small.

TABLE $\underline{43}$ Motorists' Voluntary Yielding in the Presence of Children and EIderly and Device Activation Status at the Two Study Sites

\begin{tabular}{|l|c|c|c|c|c|}
\hline Child or Elder Crossings & Total & $\begin{array}{c}\text { Lights } \\
\text { Activated }\end{array}$ & Yielding & $\begin{array}{c}\text { Lights } \\
\text { Off }\end{array}$ & Yielding \\
\hline King Avenue Site & 37 & 26 & $57.7 \%$ & 11 & $63.6 \%$ \\
Kagy Boulevard & 15 & 13 & $100.0 \%$ & 2 & $100.0 \%$ \\
\hline
\end{tabular}

\subsection{Bicyclists}

Besides pedestrians, bicyclists are relatively unique and constitute a large proportion of crosswalk users in urban areas. Table $\underline{5} 4$ provides the voluntary yielding rates in the presence and absence of bicyclists at the two study sites. 
At the King Avenue site, there were 172 crossing events by bicyclists out of the total 276 crossing events. This represents the majority of crossing events at this site: more specifically around $62 \%$. The numbers presented in this table clearly show that bicyclists are associated with different patterns than pedestrian users. Specifically, at the King Avenue site, the voluntary yielding rate for bicyclists (47.1\%) is around 75\% higher than the corresponding rate for pedestrians $(26.9 \%)$. The fact that bicyclists are more readily visible to drivers compared to pedestrians may explain the significant difference in the voluntary yielding behavior.

At the Kagy Boulevard site, bicyclists constituted a smaller proportion of sidewalk users (around $23.85 \%$ ). At this site, a different trend is illustrated in this table. Specifically, the yielding rate in the presence of bikes is $60 \%$ versus $83.3 \%$ rate in the absence of bicyclists. The relatively small number of observations with cyclist users may partly explain the different trend at this site, and as such the results should be treated with caution. The Pearson's chi-square results indicate that the presence of bicyclist users is associated with the voluntary yielding behavior at the two sites as suggested by the small P-values.

TABLE $\underline{5} 4$ Voluntary Yielding Rate in the Presence of Bicyclists at the Two Study Sites

\begin{tabular}{|l|c|c|c|c|c|c|}
\hline \multirow{2}{*}{ Bike Users? } & \multicolumn{3}{|c|}{ King Avenue } & \multicolumn{3}{c|}{ Kagy Boulevard } \\
\cline { 2 - 7 } & Yes & No & Pearson $\chi^{2}$ & Yes & No & Pearson $\chi^{2}$ \\
\hline Total Observations & 172 & 104 & & 20 & 84 & \\
Yielding & 81 & 28 & $\mathrm{P}<0.001$ & 12 & 70 & $\mathrm{P}=0.0216$ \\
Percentage & $47.1 \%$ & $26.9 \%$ & & $60.0 \%$ & $83.3 \%$ & \\
\hline
\end{tabular}

To get further insights into any potential difference in the effect of the RRFB device on the voluntary yielding behavior for the two user types, the observations were further broken down by the RRFB activation status at the King Avenue study site as shown in Table $\underline{6} 5$. The fairly small number of observations at Kagy Boulevard site may not result in reliable trends, and as such, the site was not included in this table. The yielding rates shown in this table clearly confirm the fact that the use of the RRFB device has increased the likelihood of drivers yielding to crosswalk users and that this likelihood was higher for bicyclists than that for pedestrians.

TABLE $\underline{6}$ Voluntary Yielding Rates for Bicyclists and Pedestrians with and without RRFB Activation at King Avenue Study Site

\begin{tabular}{|c|c|c|}
\hline Crosswalk Users & Bicyclists & Pedestrians \\
\hline Lights Activated & $50.5 \%$ & $32.6 \%$ \\
Lights Off & $41.0 \%$ & $22.4 \%$ \\
\hline
\end{tabular}




\subsection{Number of Crosswalk Users}

The current research attempted to examine another issue which is the potential effect of the number of crosswalk users on the motorists' voluntary yielding behavior. The hypothesis was that the larger the number of users waiting to cross at the crosswalk, the more likely for motorists to yield as they approach the crosswalk location. The rationale behind this hypothesis is that the larger number of users would make them more visible to motorists, and therefore, the motorists are expected to be more inclined to yield at the crosswalk location. Figure 4 shows the percentage of drivers yielding at the crosswalk location for different number of crosswalk users at the King Avenue study site (this analysis was only performed at King Avenue study site for sample size considerations). This figure clearly and consistently illustrates that the number of users is an important factor in determining the voluntary yielding behavior of motorists. Specifically, the larger the number of sidewalk users waiting at the crosswalk, the more likely for motorists to yield as they approach the crosswalk location. The voluntary yielding rate varied between $36.8 \%$ for single user and $80 \%$ for four or more users as shown in this figure.

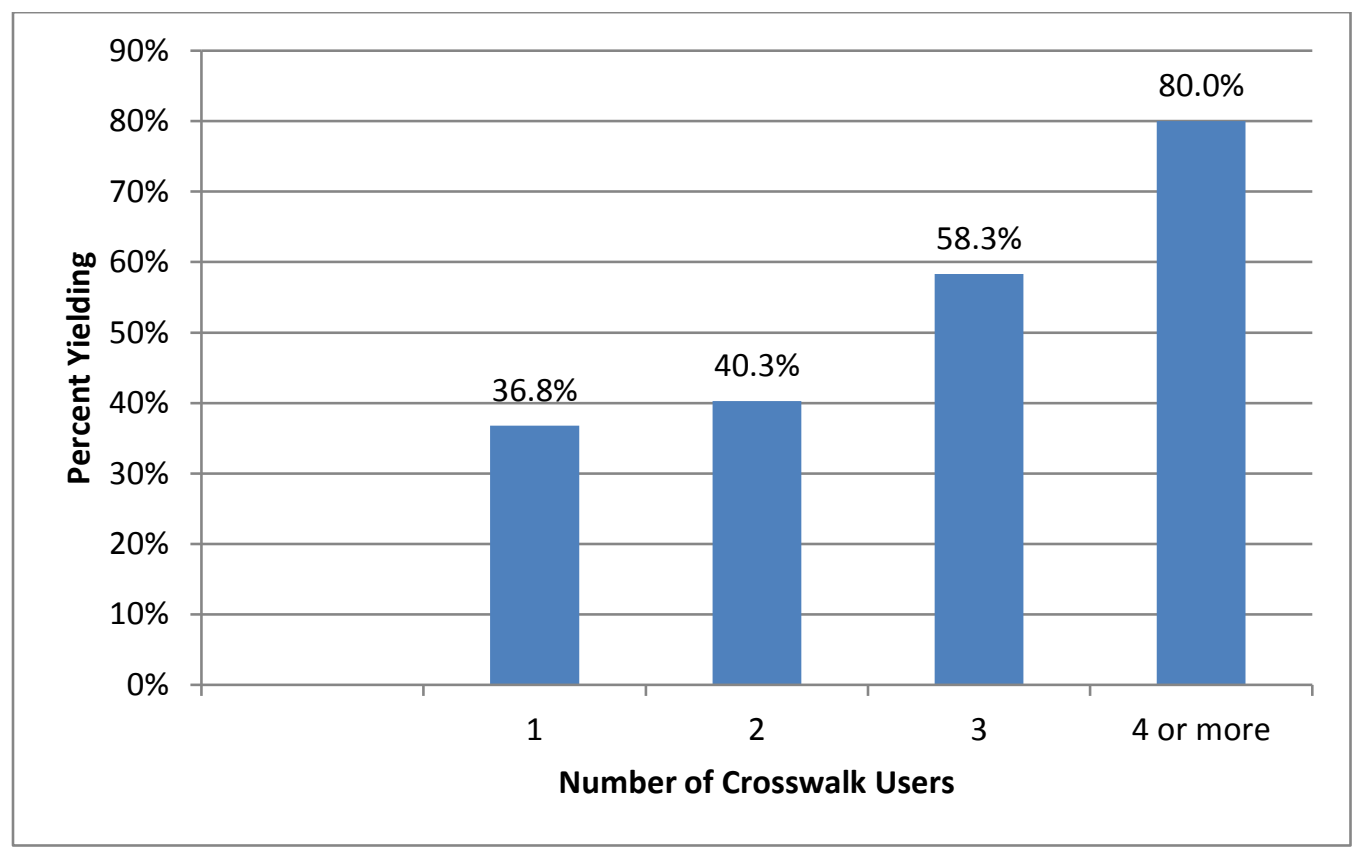

FIGURE 4 Voluntary yielding rate as a function of number of sidewalk users at King Avenue site.

To better understand the voluntary yielding behavior to pedestrians with and without device activation, the data at the King Avenue site was analyzed and results are presented in Table $\underline{7} 6$. The numbers in this table clearly show that the previous trend, i.e. the larger the number of sidewalk users, the more likely for motorists to yield as they approach the crosswalk is valid both when the device was activated as well as when it was not activated. 
TABLE $\underline{76}$ Voluntary Yielding Rates by Number of Crosswalk Users and the RRFB Activation Status at the King Avenue Study Site.

\begin{tabular}{|c|c|c|c|c|c|c|}
\hline $\begin{array}{l}\text { Number of } \\
\text { Users }\end{array}$ & Total & Yielding & Activated & Yielding & Lights Off & Yielding \\
\hline 1 & 182 & $67(36.8 \%)$ & 105 & $45(42.8 \%)$ & 77 & $22(28.5 \%)$ \\
2 & 77 & $31(40.3 \%)$ & 37 & $17(45.9 \%)$ & 40 & $14(35 \%)$ \\
3 & 12 & $7(58.3 \%)$ & 11 & $6(54.5 \%)$ & 1 & $1(100 \%)$ \\
4 or more & 5 & $4(80 \%)$ & 4 & $3(75 \%)$ & 1 & $1(100 \%)$ \\
\hline
\end{tabular}

\subsection{Peak versus Off-Peak Periods}

Another factor that could have an influence on the drivers' voluntary yielding behavior is driver characteristics and in particular whether the drivers are commuters and regular users of the highways at the study sites. This factor was investigated at the King Avenue site and results are shown in Table $\underline{87}$. The peak periods considered in this investigation are 7:00 to 9:00 in the morning and 4:00 to 6:00 in the afternoon. It was not practical to include the Kagy Boulevard site in this investigation as the number of observations during peak periods was very small.

TABLE $\underline{8} 7$ Voluntary Yielding Rates during Peak and Off-Peak Periods with and without RRFB Activation at the King Avenue Study Site

\begin{tabular}{|c|c|c|c|c|}
\hline Time Period & Activated & Yielding & Lights Off & Yielding \\
\hline Peak & 38 & $13(34.2 \%)$ & 21 & $9(42.8 \%)$ \\
Off-Peak & 119 & $58(48.7 \%)$ & 98 & $29(29.6 \%)$ \\
\hline
\end{tabular}

At a glance, it is clear that motorists yielding behavior is different during peak periods compared with the rest of daytime hours. Specifically, the yielding rate when the RRFB was activated during off-peak hours was around $49 \%$ versus only $34 \%$ during peak hours. However, an opposite trend is exhibited when the RRFB device was not activated. Another unexpected trend in this table is the higher yielding rate during peak hours when the device was not activated. While it is difficult to identify with certainty the reasons for those unexpected trends, the sample size for peak hours is relatively small (especially when the device was not activated), which may be blamed for some of the inconsistent trends found in this analysis. all crossing events that eccurred during the peak periods were associated with the RRFB device activation. One possible reason is that most drivers during peak periods may fail to yield to pedestrians, and particularly so when the RRFB device is not activated. Further, this may partly be attributed to the higher traffic levels during peak hours and the difficulty of identifying suitable gaps to finish the crossing manewvers. Another important trend presented in this table is the higher percentage of drivers yielding to pedestrian when the device was activated during off peak period compared 
with the peak period. This confirms the previous discussion about commuter drivers during peak periods being less likely to voluntarily yield to pedestrians.

\section{SUMMARY OF FINDINGS}

The current study presents an investigation into the motorists' voluntary yielding to bicyclists and pedestrians at uncontrolled mid-block crosswalk locations. Two study sites with LED RRFB warning devices in the state of Montana were used in this investigation. Video records were acquired at the two study sites and reduced using a systematic protocol to investigate the voluntary yielding behavior of motorists. The major findings of this study are:

I. Motorists' voluntary yielding increased with the activation of the RRFB devices at the two study sites. This finding is very consistent with the few recent studies that reported increase in the overall yielding behavior associated with the use of those devices.

II. Study results suggest that the willingness of motorists to voluntarily yield to bicycles and pedestrians is affected by the area and locality. The site that is located at the fringe of university campus exhibited significantly higher yielding rate compared with the other site that is located in a commercial district. However, other factors at the two sites such as cross section (two-lane undivided versus four-lane divided highways) and traffic levels may also have an effect on yielding behavior among motorists.

III. At the King Avenue site, tThe waiting position of crosswalk user relative to the roadway is found to be an important determinant of the motorists' voluntary yielding behavior. The closer the waiting position to the curb, the higher the motorists' voluntary yielding rate.

IV. The results confirmed that the motorists' voluntary yielding behavior would noticeably improve should children or elderly be among the crosswalk users. However, it should also be noted that the limited data used indicates a higher rate of RRFB activation which may partly explain the increase in motorists' yielding behavior.

V. At the King Avenue site, tThe motorists' voluntary yielding rate to bicyclists was notably higher than that to pedestrians.at the King Avenue study site, compared to that at the Kagy Boulevard site. However, the results from the latter site should be treated with eaution due to the relatively small number of observations. The number of observations at the Kagy Boulevard site was deemed too small to provide an objective validation of the aforementioned trend.

VI. Results at the King Avenue study site was very consistent in showing an increased motorist's yielding behavior with the increase in the number of users waiting at the crosswalk.

VII. During peak periods, crosswalk users are more likely to use the RRFB device. T, while his may partly be attributed to the higher traffic levels during peak hours and the 
difficulty of identifying suitable gaps to finish the crossing maneuvers.at the same time, motorists are less likely to yield during those periods.

In light of the above findings, the authors recommend that the visibility of crosswalk users by motorists be carefully considered in the selection of crosswalk locations. Further, the study recommends the push-button RRFB devices be located as close as practically possible to the roadway for increased device activation and motorists' voluntary yielding. Finally, given the limited number of sites and the size of data used in this study, the authors recommend examining other RRFB sites to get more insights into the effect of RRFB devices on motorists' voluntary yielding behavior.

Acknowledgement: The authors would like to acknowledge the financial support of the Brazil Scientific Mobility Program for funding the research assistant position.

\section{REFERENCES}

1. National Highway Transportation Safety Administration. (2014a). Traffic Safety Facts 2012 Data: Pedestrians. Publication DOT-HS-811-888. NHTSA, U.S. Department of Transportation.

2. National Highway Transportation Safety Administration. (2014b). Traffic Safety Facts 2012 Data: Bicyclists and Other Cyclists. Publication DOT-HS-812-018.NHTSA, U.S. Department of Transportation.

3. Hunter, W., Stutts, J., Pein, W., \& Cox, C. (1996). Pedestrian and Bicycle Crash Types of the Early 1990s. Publication FHWA-RD-95-163. FHWA, U.S. Department of Transportation.

4. Zegeer, C., Stewart, J., Huang, H., Lagerwey, P., Fegan, J., \& Campbell, B. (2005). Safety Effects of Marked Versus Unmarked Crosswalks at Uncontrolled Locations: Final Report and Recommended Guidelines. Publication FHWA-HRT-04-100. FHWA, U.S. Department of Transportation.

5. Ellis, R., Van Houten, R., \& Kim, J. (2007). In-Roadway "Yield to Pedestrians" Signs: Placement Distance and Driver Yielding. In Transportation Research Record: Journal of the Transportation Research Board, No.2002, Transportation Research Board of the National Academies, Washington, D.C., pp. 84-89.

6. Prevedouros, P. (2001) Evaluation of In-Pavement Flashing Lights on a Six-Lane Arterial Pedestrian Crossing. Presented at the Annual meeting of the Institute of Transportation Engineers, Chicago, IL, August 19-22, 2001.

7. Shurbutt, J., \& Van Houten, R. (2010). Effects of Yellow Rectangular Rapid Flashing Beacons on Yielding at Multilane Uncontrolled Crosswalks. Publication FHWA-HRT-10-043. FHWA, U.S. Department of Transportation, 2010.

8. Hunter, W., Srinivasan, R., \& Martell, C. (2012). Evaluation of Rectangular Rapid Flash Beacon at Pinellas Trail Crossing in Saint Petersburg, Florida. In Transportation Research Record: Journal of the Transportation Research Board, No. 2314, Transportation Research Board of the National Academies, Washington, D.C., pp. 7-13. 
9. Foster, N., Monsere, CS., \& Carlos, K. (2014). Evaluating Driver and Pedestrian Behaviors at Enhanced Multi-lane Midblock Pedestrian Crossings: A Case Study in Portland, OR. Presented at the Transportation Research Board 93rd Annual Meeting, Washington, D.C., January 12-16.

10. Hunter-Zaworski, K., \& Mueller, J. (2012). Evaluation of Alternative Pedestrian Traffic Control Devices. Publication FHWA-OR-RD-12-09. FHWA, U.S. Department of Transportation.

11. Ross, J., Serpico, D., \& Lewis, R. (2012). Assessment of Driver Yielding Rates Pre- and PostRRFB Installation, Bend, Oregon. Publication FHWA-OR-RD-12-05. FHWA, U.S. Department of Transportation, 2012.

12. Donnell, E., Hines, S., Mahoney, K., Porter, R., \& McGee, H. (2012). Speed Concepts: Informational Guide. Publication FHWA-SA-10-001. FHWA, U.S. Department of Transportation, 2012.

13. American Association of State Highway and Transportation Officials. (2011). A Policy on Geometric Design of Highways and Streets. AASHTO, Washington, DC. 